\title{
Dense Cloud Cores revealed by ALMA CO observations in the low metallicity dwarf galaxy WLM
}

\author{
M. Rubio ${ }^{1}$, B. Elmegreen ${ }^{2}$, D.Hunter ${ }^{3}$, J.Cortes ${ }^{4}$, E.Brinks ${ }^{5}$ and \\ P.Cigan ${ }^{6}$ \\ ${ }^{1}$ Departamento de Astronomia, Universidad de Chile, \\ Casilla 36-D, Santiago, Chile \\ email: mrubio@das.uchile.cl \\ ${ }^{2}$ IBM Research Division, T.J. Watson Research Center \\ NY 10598 ,USA \\ email: bge@us.ibm.com \\ ${ }^{3}$ Lowell Observatory \\ Flagstaff, Arizona 86001 ,USA \\ email: dah@lowell.edu \\ ${ }^{4}$ Joint Alma Observatory \\ Alonso de Cordova 3107, Vitacura, Chile \\ email: jcortes@alma.cl \\ ${ }^{5}$ Centre for Astrophysics Research,University of Hertfordshire \\ Hatfield AL10 9AB ,UK \\ email: e.brinks@herts.ac.uk \\ ${ }^{6}$ Cardiff University School of Physics. and Astronomy. \\ Cardiff, ,UK \\ email: pcigan1@gmail.com
}

\begin{abstract}
Understanding stellar birth requires observations of the clouds in which they form. These clouds are dense and self-gravitating, and in all existing observations, they are molecular with $\mathrm{H}_{2}$ the dominant species and $\mathrm{CO}$ the best available. When the abundances of carbon and oxygen are low compared to hydrogen, and the opacity from dust is also low, as in primeval galaxies and local dwarf irregular galaxies CO forms slowly and is easily destroyed, so it cannot accumulate inside dense clouds. Then we lose our ability to trace the gas in regions of star formation and we lose critical information on the temperatures, densities, and velocities of the material that collapses. I will report on high resolution observations with ALMA of CO clouds in the local group dwarf irregular galaxy WLM, which has a metallicity that is $13 \%$ of the solar value and $50 \%$ lower than the previous CO detection threshold and the properties derived of very small dense $\mathrm{CO}$ clouds mapped..
\end{abstract}

Keywords. molecular cloud, dwarf galaxy, low metallicity.

\section{Introduction}

Stars are formed in cold and dense molecular clouds of molecular hydrogen. Metals and molecules are essential in this process as they allow the molecular hydrogen gas to reach the temperatures required for collapse and these metals form the dust which shields the molecules from the UV radiation that destroy them. Direct observations of the $\mathrm{H}_{2}$ gas is difficult as in these cold and dense clouds, the molecular $\mathrm{H}_{2}$ does not emit radiation associated to rotational transitions. Determining the amount of molecular gas and its 
properties requires other molecular tracers and the emission of $\mathrm{CO}$ molecules is the most used one.

In particular, $\mathrm{CO}$ emission has been very difficult to detect in low metallicity galaxies. Only upper limits have been given for dwarfs galaxies with metallicities lower than the metallicity of the nearest dwarf galaxy, Small Magellanic Cloud (SMC) (Taylor et al. 1998).

The main reason why $\mathrm{CO}$ has not been detected has to do with the structure of the molecular clouds. At a given column density, or $\mathrm{A}_{v}$, the hydrogen becomes molecular and $\mathrm{CO}$ molecules can also exist. But a combination of low $\mathrm{C}$ and $\mathrm{O}$ content and less dust in dwarf galaxies attempt against survival of the CO molecules as they are less shielded and therefore suffer form photodissociation. The models of the structure of the PDR show that there are different states of $\mathrm{C}$ in layer of gas between the $\mathrm{H}_{2}$ and $\mathrm{CO}$ gas as a function of depth (Wolfire et al. 2010, Bolatto et al. 2013).

\section{WLM Breaking the metallicity barrier for CO detections at $13 \%$ of Solar metallicity}

The deployment of new sub millimeter telescope in high altitude $(5000 \mathrm{~m})$ sites in Chile equipped with sensitive receivers allowed the possibility to detect the weak and elusive CO emission from low metallicity systems. We started a program to search for $\mathrm{CO}$ and dust continuum at $350 \mathrm{GHz}$ with the APEX telescope in Chile in dwarf galaxies with lower and different metallicities than the SMC using the LITTLE THINGS survey (Hunter et al. 2012) as these galaxies had $\mathrm{H} \alpha$, HI and Spitzer $160 \mu \mathrm{m}$ data We selected WLM, an isolated dwarf galaxy located at the edge of the Local Group, at a distance is $985 \mathrm{kpc}$ with metallicty of $12+\log [\mathrm{O} / \mathrm{H}]=7.8\left(\right.$ i.e $\left.12+\log [\mathrm{O} / \mathrm{H}]_{S M C}=0.8\right)$. WLM has a gas mass of $1.6 \times 10^{7} \mathrm{M}_{\odot}$ compared to the $1.0 \times 10^{10}$ for the Milky Way (MW) and a star formation rate of $0.006 \mathrm{M}_{\odot} / \mathrm{yr}$ which is about 12 times greater than that of the MW.

The APEX observations successfuly detected CO emission for the first time in such low metallicity in two positions. Our discovery, reported in Elmegreen et al. (2013), confirmed that $\mathrm{CO}$ it is very weak in low metallicity system but showed that $\mathrm{CO}$ molecules were formed at a metallicity of only $13 \%$ solar. The CO clouds had molecular masses, determined by assuming gravitationally bound clouds, of $1.2 \times 10^{5}$ to $1.8 \times 10^{5} \mathrm{M}_{\odot}$ and surface densities of $58 \mathrm{M}_{\odot} / \mathrm{pc}^{2} 10$ times larger than the surface density found in MW clouds. We determined a conversion factor of $\alpha=124 \mathrm{M}_{\odot} \mathrm{pc}^{-2}\left(\mathrm{~K} \mathrm{~km} \mathrm{~s}^{-1}\right)^{-1}$ using the $160 \mu \mathrm{m}$ dust emission and assuming linear dependence with metallicity for the dust to gas ratio, which is consistent with the linear dependence with metallicity.

The resolution of this study was $86 \mathrm{pc}$, which is larger than most giant molecular clouds in the MilkyWay, so we could not discern the dense gas structures or internal turbulent motions and therefore we performed observations with ALMA. We observed these two WLM regions in $\mathrm{CO}(1-0)$ at arcsec resolution with ALMA in Cycle1 (Rubio et al. 2015). The observations were done with a spectral resolution of $122 \mathrm{kHz}$ per channel $(0.32$ $\mathrm{kms}^{-1}$ ) and total bandwidth of $468.750 \mathrm{MHz}$ per baseband. The $1.3 \times 0.9$ arcsec angular resolution of the observations yielded an unprecedented spatial resolution of $6.2 \mathrm{pc} \times 4.3$ pc, increasing the resolution by a factor of 18 over our previous $\mathrm{CO}(3-2)$ detections.

The delivered data were calibrated, mapped, and cleaned using the ALMA reduction software CASA. Inspecting the CO data cubes, a one channel width line was clearly seen. We re-did the cleaning (i.e., Fourier transform and beam deconvolution) using a better definition for masking of regions containing emission, and natural weighting to optimize sensitivity. The resulting data cubes have a spectral resolution of $0.5 \mathrm{kms}^{-1}$, an rms noise of $5 \mathrm{mJy}^{-1}$, and a cell size of 0.12 arcsec. CO emission could be identified from ten 
very small sized molecular clouds in both regions observed by ALMA. These resolved $\mathrm{CO}$ molecular clouds had sizes between 1 to $6 \mathrm{pc}$ revealing extremely and unexpected small sized CO clouds. Their velocity dispersions ranged from 0.6 to $1.3 \mathrm{kms}^{-1}$ and followed the size-linewidth Larson's relation for Galactic molecular clouds. Their virial masses were only a 100 to few 1000 solar masses. Their properties prove to be similar to small sized MW molecular cores but they were much denser, with surface density of 230 $\mathrm{M}_{\odot} / \mathrm{pc}^{2}$. Towards one of the WLM regions observed with ALMA, we determined that the photo-dissociation region was 5 times larger than the CO cores from CII $158 \mu \mathrm{m}$ Herschel images showing the transition between low density atomic gas and high density gas. These molecular cores are in pressure equilibrium with the weight of the overlying atomic $\mathrm{HI}$ and molecular $\mathrm{H}_{2}$ gas.

\section{Summary}

We have been able to derive for the first time the condition for molecular cloud formation in a $13 \%$ metallicty system by resolving 10 small dense molecular CO cores in WLM with sizes 25 times smaller than typical giant molecular clouds in the MW. The small mass of CO cores would imply that WLM may only form low-mass star clusters and may may explain the relative paucity of highly massive stellar clusters in small, isolated galaxies. The similarity in the WLM CO cores physical properties with galactic molecular clouds would explain why star clusters born in metal-poor galaxies resemble those seen in less-extreme systems. Also, the lack of dust in WLM means that our best tracer of $\mathrm{H}_{2}$, $\mathrm{CO}$, is present only deep in the molecular cloud making its detection difficult for lower metallicity systems. Our results show that the behavior of most of the $\mathrm{H}_{2}$ is perhaps not so different from that in other 'normal' galaxies. At only $1 \mathrm{Mpc}$ away, WLM offers an excellent opportunity to study the star formation in an extremely low metallicity environment and relate the molecular cloud properties to the cloud's environment; HI gas, dust, and star formation activity.

\section{Acknowledgements}

M.R wishes to acknowledge the SOC for the oral contribution. Her work and attendance to this IAU symposium is supported by CONICYT(CHILE) through FONDECYT grant No1140839, and partial support through BASAL PFB-06 project.

\section{References}

Bolatto, A. D., Wolfire, M., \& Leroy A. K. 2013, ARA\&A A, 51, 207-268

Elmegreen, B. G., Rubio, M., Hunter, D. A., Verdugo, C., Brinks E., \& Schruba, A. 2013, Nature, 495, 487-489

Hunter, D. A., Ficus-Vita, D., Ashley, T. et al. 2012 AJ, 144, 134

Rubio, M., Elmegreen, B. G., Hunter, D. A., Brinks E., Cortes, J., \& Cigan, P. 2015, Nature, 525,218

Wolfire, M., Hollenbach, D., \& McKee, C. F. 2010, ApJ, 716, 1191

Taylor, C., Kobulniky, H. A., \& Skillman, E. D. 1998, AJ, 116, 2747 